\title{
A QUASIREGULAR ANALOGUE OF A THEOREM OF HARDY AND LITTLEWOOD
}

\author{
CRAIG A. NOLDER
}

\begin{abstract}
Suppose that $f$ is analytic in the unit disk. A theorem of Hardy and Littlewood relates the Hölder continuity of $f$ over the unit disk to the growth of the derivative. We prove here a quasiregular analogue of this result in certain domains in $n$-dimensional space. We replace values of the derivative with a local integral average. In the process we generalize a result on the continuity of quasiconformal mappings due to Nakki and Palka. We also present another proof of the relationship between the growth of the derivative and quasiregular mappings in BMO.
\end{abstract}

Hardy and Littlewood prove the following result in [9, p. 426, Theorem 40 and p. 427, Theorem 41]. (See also [4, p. 74].)

1.1. Theorem. Suppose that $f$ is analytic in $D=\{z|| z \mid<1\}$ and $0<\alpha \leq 1$. If there exists a constant $C_{1}$ such that

$$
\left|f^{\prime}(z)\right| \leq C_{1}(1-|z|)^{\alpha-1}
$$

for all $z \in D$, then $f$ has a continuous extension to $\bar{D}=\{z|| z \mid \leq 1\}$ and

$$
\left|f\left(z_{1}\right)-f\left(z_{2}\right)\right| \leq C_{2}\left|z_{1}-z_{2}\right|^{\alpha}
$$

for all $z_{1}, z_{2} \in \bar{D}$ and some constant $C_{2}$ which depends only on $C_{1}$ and $\alpha$.

Conversely, if there exists a constant $C_{2}$ such that (1.2) holds for all $z_{1}, z_{2} \in$ $D$, then (1.1) holds for $C_{1}$ depending only on $C_{2}$ and $\alpha$.

The main result of this paper generalizes a quasiconformal version of Theorem 1.1, due to Astala and Gehring [1, Theorems 1.9 and 3.17] to a quasiregular version (Theorem 1.2) involving a somewhat larger class of moduli of continuity than $t^{\alpha}, 0<\alpha \leq 1$.

We assume throughout that $\Omega \subset R^{n}$ is an open connected set whose boundary, $\partial \Omega$, is nonempty. Also $B(x, R)$ is the open ball centered at $x \in \Omega$ with radius equal to $R>0$ and $d(x, \partial \Omega)$ is the Euclidean distance between $x$ and $\partial \Omega$. If $B \subset R^{n}$ is a ball, then $\sigma B, \sigma>0$, denotes the ball with the same center as $B$ and with radius equal to $\sigma$ times that of $B$. When $E \subset R^{n},|E|$ denotes the $n$-dimensional Lebesgue measure of $E$ and $\bar{E}$ its closure in $R^{n}$.

Received by the editors August 23, 1989 and, in revised form, February 12, 1990.

1980 Mathematics Subject Classification (1985 Revision). Primary 30C60.

Key words and phrases. Quasiregular mappings, Hölder continuity, growth of the derivative.

The work was partially supported by the National Science Foundation. 
When $f: \Omega \rightarrow R^{n}$ is differentiable, we denote its Jacobi matrix by $D f$ and the norm of the Jacobi matrix as a linear transformation by $|D f|$. When $D f$ exists a.e. we denote the local Dirichlet integral of $f$ at $x \in \Omega$ by

$$
D_{f}(x)=\left(\frac{1}{|B|} \int_{B / 2}|D f|^{n}\right)^{1 / n} .
$$

Here $B=B(x, d(x, \partial \Omega))$. When the measure is omitted from an integral, as here, integration with respect to $n$-dimensional Lebesgue measure is assumed.

A continuous increasing function $\lambda(t):[0, \infty) \rightarrow[0, \infty)$ is a majorant if $\lambda(0)=0$ and if $\lambda\left(t_{1}+t_{2}\right) \leq \lambda\left(t_{1}\right)+\lambda\left(t_{2}\right)$ for all $t_{1}, t_{2} \geq 0$.

Lip $_{\lambda}$-extension domains are a wide class of domains discussed in $\S 3$. Quasiregular mappings are discussed in $\S 2$.

We prove the following result in $\S 4$.

1.2. Theorem. Suppose that $f$ is $K$-quasiregular in a Lip $_{\lambda}$-extension domain $\Omega$. If there exists a constant $C_{1}$ such that

$$
D_{f}(x) \leq C_{1} \frac{\lambda(d(x, \partial \Omega))}{d(x, \partial \Omega)}
$$

for all $x \in \Omega$, then $f$ has a continuous extension to $\bar{\Omega}$ and

$$
\left|f\left(x_{1}\right)-f\left(x_{2}\right)\right| \leq C_{2} \lambda\left(\left|x_{1}-x_{2}\right|+d\left(x_{1}, \partial \Omega\right)\right)
$$

for all $x_{1}, x_{2} \in \bar{\Omega}$. Here $C_{2}$ is a constant which depends only on $C_{1}, K, n$, $\lambda$ and $\Omega$.

Conversely if there exists a constant $C_{2}$ such that (1.4) holds for all $x_{1}, x_{2} \in$ $\Omega$, then (1.3) holds for all $x \in \Omega$ with $C_{1}$ depending only on $C_{2}, K, n, \lambda$ and $\Omega$.

If $f$ is analytic and if $\lambda(t)=t^{\alpha}$, then the conditions (1.1) and (1.3) are equivalent.

Simple examples show that the term $d\left(x_{1}, \partial \Omega\right)$ cannot in general be omitted. For example $f(x)=x|x|^{a-1}$ with $a=K^{1 /(1-n)}$ is $K$-quasiconformal in $B=$ $B(0,1), D_{f}(x)$ is bounded over $x \in B$ yet $f \in \operatorname{Lip}_{a}(B)$ (see [1, Remark 3.12]). However, by suitably modifying a theorem of Näkki and Palka [13] to the quasiregular case, we obtain the following result.

1.3. Theorem. Suppose that $f$ is $K$-quasiregular in a Lip $_{\lambda}$-extension domain $\Omega$ where $\lambda(t)=t^{\alpha}$ and $0<\alpha \leq K^{1 /(1-n)}$.

If there exists a constant $C_{1}$ such that

$$
D_{f}(x) \leq C_{1} d(x, \partial \Omega)^{\alpha-1}
$$

for all $x \in \Omega$, then $f$ has a continuous extension to $\bar{\Omega}$ and

$$
\left|f\left(x_{1}\right)-f\left(x_{2}\right)\right| \leq C_{2}\left|x_{1}-x_{2}\right|^{\alpha}
$$

for all $x_{1}, x_{2} \in \bar{\Omega}$.

Here $C_{2}$ depends only on $C_{1}, K, n, \alpha$, and $\Omega$.

The converse follows from Theorem 1.2. 
In the case that $f$ is quasiconformal, Astala and Gehring prove Theorem 1.2, with $\lambda(t)=t^{\alpha}$, and Theorem 1.3 with the operator $a_{f}(x)$ in place of $D_{f}(x)$ [1, Theorems 1.9, 3.17, and 3.13]. Here, with $B=B(x, d(x, \partial \Omega))$,

$$
a_{f}(x)=\exp \left\{\frac{1}{n|B|} \int_{B} \log J_{f}\right\}
$$

where $J_{f}$ is the determinant of the Jacobi matrix $D f$. When $f$ is quasiconformal, $\log J_{f}$ is integrable over each ball $B \subset \Omega$. If $n=2$ and $f$ is conformal, then $\log J_{f}$ is harmonic and $a_{f}(x)=\left|f^{\prime}(x)\right|$. When $f$ is quasiconformal $a_{f}(x)$ and $D_{f}(x)$ are equivalent (Lemma 2.7) and because of this, Theorems 1.2 and 1.3 reduce to the results in [1]. As the example $z^{n}$ shows, the ratio $D_{f}(x) / a_{f}(x)$ may depend on the local topological index and is in general unbounded for quasiregular $f$.

We also prove a result corresponding to Theorem 1.2 in the case $\alpha=0$. Although Theorem 1.4 follows from a result of Vuorinen [18, p. 104, Theorem 4.29] the proof given here is similar to the proof of Theorem 1.2.

If $f=\left(f_{1}, f_{2}, \ldots, f_{n}\right): \Omega \rightarrow R^{n}$, then we write, with $B=B(x, d(x, \partial \Omega))$,

$$
D_{f_{j}}(x)=\left(\frac{1}{|B|} \int_{B / 2}\left|\nabla f_{j}\right|^{n}\right)^{1 / n} \text {. }
$$

We write $\|f\|_{*}$ for the BMO norm of $f$ over $\Omega$ (see $\S 6$ ).

1.4. Theorem. Suppose that $f=\left(f_{1}, f_{2}, \ldots, f_{n}\right)$ is $K$-quasiregular in $\Omega$. If there exists a constant $C_{1}$ so that

$$
D_{f_{j}}(x) \leq C_{1} d(x, \partial \Omega)^{-1}
$$

for some $1 \leq j \leq n$ and all $x \in \Omega$, then

$$
\left\|f_{j}\right\|_{*} \leq C_{2}<\infty
$$

where $C_{2}$ depends only on $C_{1}, K$, and $n$.

Conversely, if (1.6) holds for some constant $C_{2}$ and some $1 \leq j \leq n$, then (1.5) holds with $C_{1}$ depending only on $C_{2}, K$, and $n$.

Theorem 1.4 gives a simple proof of Corollary 3 in [10, p. 280], which states that the BMO-norms of the components of a quasiregular mapping are equivalent.

2.1. Quasiregular mappings. We denote by $W_{n}^{1}(\Omega)$ the Sobolev space of functions $f: \Omega \rightarrow R^{m}$ which are $L^{n}$-integrable over $\Omega$ and have $L^{n}$-integrable distributional first derivatives over $\Omega . W_{n, \text { loc }}^{1}(\Omega)=\bigcap W_{n}^{1}\left(\Omega^{\prime}\right)$ where the intersection is over all $\Omega^{\prime}$ compactly contained in $\Omega$.

2.2. Definition. A function $f: \Omega \rightarrow R^{n}$ is $K$-quasiregular in $\Omega \subset R^{n}, 1 \leq$ $K<\infty$, if

(a) $f \in W_{n, \text { loc }}^{1}(\Omega)$,

(b) $|D f|^{n} / K \leq J_{f} \leq K l(D f)^{n}$ a.e. in $\Omega$ where $l(D f)=\inf \{|D f s||| s \mid=1\}$.

When $n=2, f$ is 1-quasiregular if and only if it is an analytic function. A homeomorphism in $R^{n}$ is quasiregular if and only if it is quasiconformal in the usual sense. For information on quasiregular mappings see [3,12, and 20].

We list here some preliminary results. 
2.3. Proposition. Suppose that $B \subset R^{n}$ is a ball and $s>n$. If $f \in W_{s}^{1}(B)$, then there is a constant $C$, depending only on $n$, such that

$$
\left|f\left(x_{1}\right)-f\left(x_{2}\right)\right| \leq \frac{C_{s}}{s-n}|B|^{(s-n) / s n}\left(\int_{B}|D f|^{s}\right)^{1 / s}
$$

for all $x_{1}, x_{2} \in B$.

A proof of Proposition 2.3 can be found in [3, p. 268, Lemma 1.7].

2.4. Proposition. If $f \in W_{n}^{1}(B)$, where $B \subset R^{n}$ is a ball, then

$$
\left(\frac{1}{|B|} \int_{B}\left|f-f_{B}\right|^{n}\right)^{1 / n} \leq 2 \operatorname{diam} B\left(\frac{1}{|B|} \int_{B}|D f|^{n}\right)^{1 / n} .
$$

Here $\operatorname{diam} B$ is the Euclidean diameter of $B$ and $f_{B}$ is the average value of $f$ over $B$.

This result is a special case of Lemma 1.5, p. 266, in [3].

Proposition 2.5, which appears in [3, p. 285, Theorem 5.1], shows that Proposition 2.3 can be applied to quasiregular mappings. For quasiconformal mappings Proposition 2.5 is due to Gehring [7, p. 274, Theorem 1].

2.5. Proposition. If $f$ is $K$-quasiregular in $\Omega$, then there are constants, $s>n$ and $C<\infty$, which depend only on $n$ and $K$, such that $f \in W_{s, \mathrm{loc}}^{1}(\Omega)$ and

$$
\left(\int_{F}|D f|^{s}\right)^{1 / s} \leq C d(F, \partial \Omega)^{(n-s) / s}\left(\int_{\Omega}|D f|^{n}\right)^{1 / n}
$$

for all compact sets $F \subset \Omega$. Here $d(F, \partial \Omega)$ is the Euclidean distance between $F$ and $\partial \Omega$.

A proof of the next result can be found in [10, p. 277, (5.3)].

2.6. Proposition. If $f=\left(f_{1}, f_{2}, \ldots, f_{n}\right)$ is $K$-quasiregular in $\Omega$ and if $B$ is a ball with $\sigma B \subset \Omega, \sigma>1$, then there exists a constant $C$, depending only on $n$, such that

$$
\left(\int_{B}|D f|^{n}\right)^{1 / n} \leq C K \frac{\sigma}{\sigma-1}\left(\frac{1}{|\sigma B|} \int_{\sigma B}\left|f_{j}-a\right|^{n}\right)^{1 / n}
$$

for all $a \in R$ and all $j=1,2, \ldots, n$.

The following lemma shows that Theorem 1.2 is equivalent to the result of Astala and Gehring [1, Theorems 1.9 and 3.7] if $f$ is quasiconformal and $\lambda(t)=t^{\alpha}$.

2.7. Lemma. If $f$ is $K$-quasiconformal in $\Omega$, then there exists a constant $C$, which depends only on $n$ and $K$, such that

$$
\frac{1}{C} a_{f}(x) \leq D_{f}(x) \leq C a_{f}(x)
$$

for all $x \in \Omega$.

Proof. Since $f$ is quasiconformal, $\left\|\log J_{f}\right\|_{*}<\infty$ [15, p. 261, Theorem 1]. The John-Nirenberg Theorem [15, p. 260, Lemma 1] and [5, p. 230, Theorem 
2.1] implies that there exist positive constants $a, b$, and $C_{1}$, which depend only on $n$ and the BMO-norm $\left\|\log J_{f}\right\|_{*}$, so that

$$
\left(\frac{1}{|R|} \int_{R} J_{f}^{a}\right)^{1 / a} \leq C_{1}\left(\frac{1}{|R|} \int_{R} J_{f}^{-b}\right)^{-1 / b}
$$

for all balls $R$ with $\bar{R} \subset \Omega$ [15, p. 263, Lemma 3]. Next, by a result of Gehring [7, p. 271, Lemma 4], there exists a constant $C_{2}$, depending only on $n$ and $K$, such that

$$
\left(\frac{1}{|R|} \int_{R}|D f|^{n}\right)^{1 / n} \leq C_{2}\left(\frac{1}{|R|} \int_{R}|D f|\right)
$$

for all balls $R$ with $\bar{R} \subset \Omega$. It follows from Hölder's inequality (see [10, Remark on p. 272] that we can improve the exponent in the reverse Hölder inequality (2.6) to obtain

$$
\left(\frac{1}{|R|} \int_{R}|D f|^{n}\right)^{1 / n} \leq C_{3}(n, r)\left(\frac{1}{|R|} \int_{R}|D f|^{r}\right)^{1 / r}
$$

for all balls $R$ with $\bar{R} \subset \Omega$ and all $r, 0<r \leq n$. Fix $x \in \Omega$ and let $B=B\left(x, \frac{1}{2} d(x, \partial \Omega)\right)$. Using the dilatation inequality Definition 2.2(a), (2.7) with $r=n a$ and (2.5) we obtain

$$
\begin{aligned}
& \left(\frac{1}{|B|} \int_{B}|D f|^{n}\right)^{1 / n} \leq C_{4}\left(\frac{1}{|B|} \int_{B}|D f|^{n a}\right)^{1 /(n a)} \\
& \quad \leq C_{4} K^{1 / n}\left(\frac{1}{|B|} \int_{B} J_{f}^{a}\right)^{1 /(n a)} \leq C_{5}\left(\frac{1}{|B|} \int_{B} J_{f}^{-b}\right)^{-1 /(n b)} .
\end{aligned}
$$

From Jensen's inequality for convex functions [5, p. 34] we obtain

$$
\left(\frac{1}{|2 B|} \int_{2 B} J_{f}^{-b}\right)^{-1 / b} \leq \exp \left(\frac{1}{|2 B|} \int_{2 B} \log J_{f}\right) .
$$

If $\|u\|_{*}<\infty$ and $B_{0}$ and $B_{1}$ are balls with $B_{0} \subset B_{1} \subset \Omega$, then

$$
\left|\frac{1}{\left|B_{0}\right|} \int_{B_{0}} u-\frac{1}{\left|B_{1}\right|} \int_{B_{1}} u\right| \leq \frac{e}{2}\left(\log \frac{\left|B_{0}\right|}{\left|B_{1}\right|}+1\right)\|u\|_{*} \text {. }
$$

See Lemma 5.10 in [2]. Combining (2.8) and (2.9) and applying (2.10) with $B_{0}=B, B_{1}=2 B$ and $u=\log J_{f}$ we obtain

$$
D_{f}(x) \leq \exp \left(\frac{e}{2 n}(n \log 2+1)\left\|\log J_{f}\right\|_{*}\right) C_{5} a_{f}(x) .
$$

Next using the inequality $J_{f} \leq|D f|^{n}$ a.e. and Jensen's inequality we obtain

$$
\exp \left(\frac{1}{n|B|} \int_{B} \log J_{f}\right) \leq D_{f}(x)
$$

Combining (2.10) and (2.11),

$$
a_{f}(x) \leq \exp \left(\frac{e}{2 n}(n \log 2+1)\left\|\log J_{f}\right\|_{*}\right) D_{f}(x) .
$$

3.1. $\operatorname{Lip}_{\lambda}$-classes and $\operatorname{Lip}_{\lambda}$-extension domains. Suppose that $f: E \rightarrow R^{m}$, $E \subset R^{n}$. If there exists a constant $C$ such that

$$
\left|f\left(x_{1}\right)-f\left(x_{2}\right)\right| \leq C \lambda\left(\left|x_{1}-x_{2}\right|\right)
$$


for all $x_{1}, x_{2} \in E$, then we write $f \in \operatorname{Lip}_{\lambda}(E)$. We denote the smallest such $C$ by $\|f\|_{\lambda}$. If $\lambda(t)=t^{\alpha}$ we write $\operatorname{Lip}_{\alpha}(E)$ and $\|f\|_{\alpha}$. If there exists a constant $C$ such that

$$
\left|f\left(x_{1}\right)-f\left(x_{2}\right)\right| \leq C \lambda\left(\left|x_{1}-x_{2}\right|+d\left(x_{1}, \partial \Omega\right)\right)
$$

for all $x_{1}, x_{2} \in E$, then we write $f \in \operatorname{Lip}_{\lambda}^{*}(E)$ and denote the smallest such $C$ by $\|f\|_{\lambda}^{*}$. We similarly define $\operatorname{Lip}_{\alpha}^{*}(E)$ and $\|f\|_{\alpha}^{*}$.

If (3.1) holds for all $x_{1}, x_{2} \in \Omega$ with $\left|x_{1}-x_{2}\right| \leq \frac{1}{2} d\left(x_{1}, \partial \Omega\right)$ we write $f \in \operatorname{loc}_{\operatorname{Lip}_{\lambda}(\Omega)}$ and $\|f\|_{\lambda, \text { loc }}$. Similarly for (3.2) we write $f \in \operatorname{loc}_{\operatorname{Lip}}^{*}(\Omega)$ and $\|f\|_{\lambda, \text { loc }}^{*}$. In [11, Theorem 2.17] Lappalainen shows that if (3.1) holds whenever $\left|x_{1}-x_{2}\right| \leq a d\left(x_{1}, \partial \Omega\right)$ for some $a \leq 1$, then $f \in \operatorname{loc}_{\operatorname{Lip}}(\Omega)$. The proof shows that a similar result also holds for $\operatorname{loc}_{\operatorname{Lip}}^{*}(\Omega)$.

3.2. Definition. $\Omega \subset R^{n}$ is a $\operatorname{Lip}_{\lambda}$-extension domain if $f \in \operatorname{Lip}_{\lambda}(\Omega)$ whenever $f \in \operatorname{loc} \operatorname{Lip}_{\lambda}(\Omega)$. In other words there exists a constant $b$ such that $\|f\|_{\lambda} \leq$ $b\|f\|_{\lambda, \text { loc }}$ for all $f: \Omega \rightarrow R^{m}$.

A domain $\Omega$ is a $\operatorname{Lip}_{\lambda}$-extension domain if and only if there exists a constant $M$ such that each pair $x_{1}, x_{2} \in \Omega$ can be joined by a continuous curve $\gamma \subset \Omega$ which satisfies

$$
\int_{\gamma} \frac{\lambda(d(\gamma(s), \partial \Omega))}{d(\gamma(s), \partial \Omega)} d s \leq M \lambda\left(\left|x_{1}-x_{2}\right|\right)
$$

(see [11, p. 23, Theorem 4.2]). Here $d s$ is the element of arclength. These domains were first introduced by Gehring and Martio in the case that $\lambda(t)=$ $t^{\alpha}$ and called $\operatorname{Lip}_{\alpha}$-extension domains. For certain $\lambda(t)$, the class of $\operatorname{Lip}_{\lambda^{-}}$ extension domains is large. All uniform domains are Lip $_{\lambda}$-extension domains if and only if there is a constant $A$ such that

$$
\int_{0}^{\delta} \frac{\lambda(t)}{t} d t \leq A \lambda(\delta)
$$

for all $0 \leq \delta<\infty$ (see [11, p. 28, Theorem 4.17] and [8, p. 204, Theorem 2.2]. In particular, if $\lambda(t)=t^{\alpha}$, all balls, half-spaces and quasiballs are $\operatorname{Lip}_{\alpha^{-}}$ extension domains for all $0<\alpha \leq 1$.

3.3. Lemma. If $\Omega$ is a $\operatorname{Lip}_{\lambda}$-extension domain and if $f \in \operatorname{loc}_{\operatorname{Lip}}^{*}(\Omega)$, then $f \in \operatorname{Lip}_{\lambda}^{*}(\Omega)$.

The proof is similar to the proof of the characterization of $\operatorname{Lip}_{\alpha}$-extension domains in [8] and $\mathrm{Lip}_{\lambda}$-extension domains in [11]. We include the proof here for completeness.

Proof. Fix $x_{1}, x_{2} \in \Omega$ and let $\gamma$ be a curve joining $x_{1}$ to $x_{2}$ in $\Omega$ which satisfies (3.3). Choose balls $B\left(y_{i}, r_{i}\right)=\left\{x|| x-y_{i} \mid<r_{i}\right\}$ as follows. Set $y_{1}=x_{2}, r_{1}=\frac{1}{4} d\left(y_{1}, \partial \Omega\right)$ and $l_{1}=\max \left\{s \in[0, l] \mid \gamma(s) \in \bar{B}\left(y_{1}, r_{1}\right)\right\}$ where $l$ is the length of $\gamma$. Suppose that $y_{i}, r_{i}$ and $l_{i}$ have been chosen for $i=$ $1,2, \ldots, k$ and $l_{k}<l$. Set $y_{k+1}=\gamma\left(l_{k}\right), r_{k+1}=\frac{1}{4} d\left(y_{k+1}, \partial \Omega\right)$ and $l_{k+1}=$ $\max \left\{s \in[0, l] \mid \gamma(s) \in \bar{B}\left(y_{k+1}, r_{k+1}\right)\right\}$. When $l_{k}=l$, the process stops and we write $y_{k+1}=x_{1}$. 
First suppose that $\left|x_{1}-x_{2}\right|<\left|y_{k}-y_{k+1}\right|=\left|y_{k}-x_{1}\right|$. Then $\left|x_{1}-x_{2}\right| \leq$ $\frac{1}{4} d\left(y_{k}, \partial \Omega\right)$. Hence

$$
d\left(x_{1}, \partial \Omega\right) \geq d\left(y_{k}, \partial \Omega\right)-\left|x_{1}-y_{k}\right| \geq \frac{3}{4} d\left(y_{k}, \partial \Omega\right)
$$

and so $\left|x_{1}-x_{2}\right| \leq \frac{1}{3} d\left(x_{1}, \partial \Omega\right)$. Since $f \in \operatorname{loc}_{\operatorname{Lip}_{\lambda}^{*}}^{*}(\Omega)$,

$$
\left|f\left(x_{1}\right)-f\left(x_{2}\right)\right| \leq\|f\|_{\lambda, \text { loc }}^{*} \lambda\left(\left|x_{1}-x_{2}\right|+d\left(x_{1}, \partial \Omega\right)\right) .
$$

Hence we can suppose that

$$
\left|y_{k}-y_{k+1}\right| \leq\left|x_{1}-x_{2}\right| \text {. }
$$

Next

$$
\begin{aligned}
\left|f\left(x_{1}\right)-f\left(x_{2}\right)\right| & \leq \sum_{i=1}^{k}\left|f\left(y_{i}\right)-f\left(y_{i+1}\right)\right| \\
& \leq\|f\|_{\lambda, \text { loc }}^{*} \sum_{i=1}^{k} \lambda\left(\left|y_{i}-y_{i+1}\right|+d\left(y_{i}, \partial \Omega\right)\right) .
\end{aligned}
$$

Let $l_{0}=0$ and set $A_{i}=\left\{s \in\left[l_{i-1}, l_{i}\right] \mid \gamma(s) \in \bar{B}\left(y_{i}, r_{i}\right)\right\}$ for $i=1,2, \ldots$, $k-1 . A_{i} \subset\left[l_{i-1}, l_{i}\right]$ is a closed set and $\left|A_{i}\right| \geq r_{i}=\left|y_{i}-y_{i+1}\right|$. Here $\left|A_{i}\right|$ is the one-dimensional Lebesgue measure of $A_{i}$. Also for each $s \in A_{i}$,

$$
d(\gamma(s), \partial \Omega) \leq\left|\gamma(s)-y_{i}\right|+d\left(y_{i}, \partial \Omega\right) \leq r_{i}+4 r_{i}=5 r_{i}
$$

Hence, since $\lambda(t) / t$ is decreasing,

$$
\frac{\lambda\left(5 r_{i}\right)}{r_{i}} \leq \frac{5 \lambda(d(\gamma(s), \partial \Omega))}{d(\gamma(s), \partial \Omega)}
$$

when $s \in A_{i}$. Using (3.5), (3.4), (3.6), and (3.3) we obtain

$$
\begin{aligned}
& \left|f\left(x_{1}\right)-f\left(x_{2}\right)\right| \leq\|f\|_{\lambda, \text { loc }}^{*} \sum_{i=1}^{k} \lambda\left(\left|y_{i}-y_{i+1}\right|+d\left(y_{i}, \partial \Omega\right)\right) \\
& \leq\|f\|_{\lambda, \text { loc }}^{*}\left\{\sum_{i=1}^{k-1} \lambda\left(5\left|y_{i}-y_{i+1}\right|\right)+\lambda\left(\left|y_{k}-y_{k+1}\right|+d\left(y_{k}, \partial \Omega\right)\right)\right\} \\
& \leq\|f\|_{\lambda, \operatorname{loc}}^{*}\left\{\sum_{i=1}^{k-1} \frac{\lambda\left(5 r_{i}\right)}{r_{i}}\left|A_{i}\right|+\lambda\left(2\left|x_{1}-x_{2}\right|+d\left(x_{1}, \partial \Omega\right)\right)\right\} \\
& \leq\|f\|_{\lambda, \text { loc }}^{*}\left\{5 \sum_{i=1}^{k-1} \int_{A_{i}} \frac{\lambda(d(\gamma(s), \partial \Omega))}{d(\gamma(s), \partial \Omega)} d s+2 \lambda\left(\left|x_{1}-x_{2}\right|+d\left(x_{1}, \partial \Omega\right)\right)\right\} \\
& \leq 5\|f\|_{\lambda, \text { loc }}^{*}\left\{\int_{\gamma} \frac{\lambda(d(\gamma(s), \partial \Omega))}{d(\gamma(s), \partial \Omega)} d s+\lambda\left(\left|x_{1}-x_{2}\right|+d\left(x_{1}, \partial \Omega\right)\right)\right\} \\
& \leq 5 M\|f\|_{\lambda, \text { loc }}^{*} \lambda\left(\left|x_{1}-x_{2}\right|+d\left(x_{1}, \partial \Omega\right)\right) \text {. }
\end{aligned}
$$

4.1. Proof of Theorem 1.2. First suppose that (1.3) holds for all $x \in \Omega$. Since $f$ is $K$-quasiregular there exists $s>n$, depending only on $n$ and $K$, such that 
$f \in W_{s, \text { loc }}^{1}(\Omega)$. Fix $x_{1}, x_{2} \in \Omega$ with $\left|x_{1}-x_{2}\right|=\frac{1}{4} d\left(x_{1}, \partial \Omega\right)$. We apply (2.1) with $B=B\left(x_{1}, \frac{1}{4} d\left(x_{1}, \partial \Omega\right)\right)$, and (2.3) with $F=\bar{B}$ and $\Omega=2 B$ to obtain

$$
\begin{aligned}
\left|f\left(x_{1}\right)-f\left(x_{2}\right)\right| & \leq \frac{C s}{s-n}|B|^{(s-n) /(s n)}\left(\int_{B}|D f|^{s}\right)^{1 / s} \\
& \leq C_{1}(n, K)\left(\int_{2 B}|D f|^{n}\right)^{1 / n} \\
& \leq C_{1}(n, K) C_{2}\left|x_{1}-x_{2}\right| D_{f}\left(x_{1}\right) \\
& \leq 4 C_{1}(n, K) C_{2} \lambda\left(d\left(x_{1}, \partial \Omega\right)\right) \\
& \leq 16 C_{1}(n, K) C_{2} \lambda\left(\left|x_{1}-x_{2}\right|\right) .
\end{aligned}
$$

Now assume that $\left|x_{1}-x_{2}\right|<\frac{1}{4} d\left(x_{1}, \partial \Omega\right)$. Let $R_{1}=\frac{1}{4} d\left(x_{1}, \partial \Omega\right)$ and $R_{2}=\frac{1}{4} d\left(x_{2}, \partial \Omega\right)$. Now $R_{2} \leq R_{1}+\frac{1}{4}\left|x_{1}-x_{2}\right| \leq \frac{5}{4} R_{1}$ and $R_{2} \geq R_{1}-$ $\frac{1}{4}\left|x_{1}-x_{2}\right| \geq R_{1}-\left|x_{1}-x_{2}\right|$. Hence there exists a point $x_{3}$ with $x_{3} \in$ $\partial B\left(x_{1}, R_{1}\right) \cap \partial B\left(x_{2}, R_{2}\right)$. So we obtain $\left|f\left(x_{1}\right)-f\left(x_{2}\right)\right| \leq\left|f\left(x_{1}\right)-f\left(x_{3}\right)\right|+$ $\left|f\left(x_{2}\right)-f\left(x_{3}\right)\right| \leq C\left(\lambda\left(\left|x_{1}-x_{3}\right|\right)+\lambda\left(\left|x_{2}-x_{3}\right|\right)\right)$ by applying (4.1) to the pairs $x_{1}, x_{3}$ and $x_{2}, x_{3}$. It follows that

$$
\left|f\left(x_{1}\right)-f\left(x_{2}\right)\right| \leq C\left(\lambda\left(d\left(x_{1}, \partial \Omega\right)\right)+\lambda\left(\left|x_{1}-x_{2}\right|+d\left(x_{1}, \partial \Omega\right)\right)\right)
$$

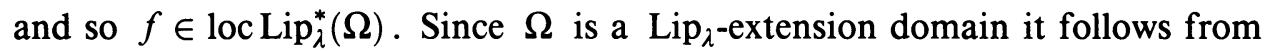
Lemma 3.3 that $f \in \operatorname{Lip}_{\lambda}^{*}(\Omega)$. Next if $x_{0} \in \partial \Omega$ and $\left\{x_{j}\right\}$ is a sequence in $\Omega$ with $\lim _{j \rightarrow \infty} x_{j}=x_{0}$ we have

$$
\left|f\left(x_{j}\right)-f\left(x_{k}\right)\right| \leq C \lambda\left(\left|x_{j}-x_{k}\right|+d\left(x_{j}, \partial \Omega\right)\right) .
$$

Hence $f$ tends to a well-defined limit at $x_{0}$ and (1.4) is satisfied for all $x_{1}, x_{2} \in$ $\bar{\Omega}$.

Next suppose that (1.4) holds for all $x_{1}, x_{2} \in \bar{\Omega}$. Fix $x \in \Omega$. We apply (2.3) with $B=B\left(x, \frac{1}{2} d(x, \partial \Omega)\right), \sigma=\frac{3}{2}$ and $a=f(x)$ to obtain

$$
D_{f}(x) \leq \frac{C_{1}(n) K}{d(x, \partial \Omega)}\left(\frac{1}{|B|} \int_{3 B / 2}|f-f(x)|^{n}\right)^{1 / n} .
$$

Using (1.4) we get

$$
\begin{aligned}
& \left(\frac{1}{|B|} \int_{3 B / 2}|f-f(x)|^{n}\right)^{1 / n} \leq \frac{3}{2} \lambda\left(\sup _{y \in 3 B / 2}|x-y|+d(x, \partial \Omega)\right) \\
& \quad \leq \frac{3}{2} \lambda(2 d(x, \partial \Omega)) \leq 3 \lambda(d(x, \partial \Omega)) .
\end{aligned}
$$

(1.3) follows by combining (4.1) and (4.2).

5.1. Proof of Theorem 1.3. We use the following definitions and results. A pair of sets $E=(A, C)$ in $R^{n}$ is called a condenser when $A$ is open and $C \subset A$ is compact. The condenser $E$ is bounded if $A$ is bounded. Its conformal capacity is defined by

$$
\operatorname{cap} E=\inf _{u} \int_{R^{n}}|\nabla u|^{n}
$$


where the infimum is taken over all infinitely differentiable $u$ in $A$ with compact support in $A$ and $u(x) \geq 1$ for $x \in C$. If follows from the definition that if $A^{\prime} \subset A$ and $C \subset C^{\prime}$, then

$$
\operatorname{cap}(A, C) \leq \operatorname{cap}\left(A^{\prime}, C^{\prime}\right) \text {. }
$$

For more information concerning condensers see [12, pp. 24-28; 20, pp. 81102 and 17]. We next define the spherical symmetrization, $E^{*}$, of an open or closed set $E \subset R^{n}$, about the ray $L=\{t \xi \mid 0 \leq t<\infty, \xi$ some point of $\partial B(0,1)\}$ as follows. We let $S^{n-1}(r)=\partial B(0, r)$. We call sets of the form $B(y, \rho) \cap S^{n-1}(r)$, with $y \in S^{n-1}(r)$ and $0 \leq \rho<\infty$, caps of $S^{n-1}(r)$. We define $E^{*}$ by the conditions:

$$
S^{n-1}(r) \cap E^{*}= \begin{cases}\varnothing, & \text { if and only if } S^{n-1}(r) \cap E=\varnothing, \\ S^{n-1}(r), & \text { if and only if } S^{n-1}(r) \subset E .\end{cases}
$$

Otherwise, $S^{n-1}(r) \cap E^{*}$ is the cap of the sphere $S^{n-1}(r)$ such that

(i) the center of the cap is the point $S^{n-1}(r) \cap L$.

(ii) $m_{n-1}\left(S^{n-1}(r) \cap E^{*}\right)=m_{n-1}\left(S^{n-1}(r) \cap E\right)$. Here $m_{n-1}$ is the $(n-1)$ dimensional Hausdorff measure on $S^{n-1}(r)$.

(iii) the cap $S^{n-1}(r) \cap E^{*}$ is open or closed according as $E$ is open or closed. Here $0 \leq r<\infty$. For the following result see Gehring [6, p. 505, Theorem 1] and Sarvas [17, p. 522, Theorem 7.5].

5.2. Theorem. If $E=(A, C)$ is a condenser let $C^{*}$ be the spherical symmetrization of $C$ about the negative $x_{1}$-axis and $A^{*}=R^{n} \backslash B$ where $B$ is the spherical symmetrization of $R^{n} \backslash A$ in the positive $x_{1}$-axis, then $\left(A^{*}, C^{*}\right)$ is a condenser and

$$
\operatorname{cap}\left(A^{*}, C^{*}\right) \leq \operatorname{cap}(A, C) .
$$

Theorem 1.3 follows from Theorem 1.2 and the next result. The quasiconformal version of Theorem 5.3 appears in Näkki and Palka [13, p. 379, Theorem 1]. Our proof is similar to their proof.

5.3. Theorem. Suppose that $f$ is $K$-quasiregular in $\Omega \subset R^{n}$ and continuous on $\bar{\Omega}$. If there exist constants $\alpha, 0<\alpha \leq K^{1 /(1-n)}$, and $C_{1}<\infty$ such that

$$
|f(x)-f(y)| \leq C_{1}|x-y|^{\alpha}
$$

for all $x \in \bar{\Omega}$ and $y \in \partial \Omega$, then $f \in \operatorname{Lip}_{\alpha}(\bar{\Omega})$ with $\|f\|_{\alpha}$ depending only on $C_{1}, n, K$, and $\alpha$. If $\Omega$ is bounded and (5.2) holds for some $0<\alpha \leq 1$, then $f \in \operatorname{Lip}_{\beta}(\bar{\Omega})$ with $\beta=\min \left(\alpha, K^{1 /(1-n)}\right)$.

Proof. Assume that $f$ is not constant. Fix $x$ and $y$ with $f(x) \neq f(y)$. First suppose that $|x-y| \geq \frac{1}{2} d(x, \partial \Omega)$. Choose $z \in \partial \Omega$ such that $|x-z|=$ $d(x, \partial \Omega)$. Using $(5.2)$ we obtain

$$
|f(x)-f(y)| \leq|f(x)-f(z)|+|f(y)-f(z)| \leq 6^{\alpha} C_{1}|x-y|^{\alpha} .
$$

Next suppose that $|x-y|<d(x, \partial \Omega) / 2$. Let $B=B(x, d(x, \partial \Omega) / 2)$ and $C=\bar{B}(x,|x-y|)$. Then $E=(B, C)$ is a bounded condenser with capacity

$$
\operatorname{cap} E=\omega_{n-1}\left(\log \frac{d(x, \partial \Omega)}{2|x-y|}\right)^{1-n} .
$$


Here $\omega_{n-1}$ is the $(n-1)$-dimensional measure of $\partial B(0,1)$. Since $f$ is quasiregular and nonconstant, $f$ is continuous and open in $\Omega$. Hence $F=$ $(f(B), f(C))$ is a bounded condenser in $f(\Omega)$ and $\partial f(B) \subset f(\partial B)$. Since $f(B)$ is bounded, $R^{n} \backslash f(B)$ contains a, necessarily unique, unbounded component $C_{\infty}$ with $\partial C_{\infty} \neq \varnothing$. Let $\xi \in \partial C_{\infty}$. Since $\partial C_{\infty} \subset \partial f(B), \xi \in \partial f(B)$. Since $\partial f(B) \subset f(\partial B)$, there is a point $z \in \partial B$ such that $f(z)=\xi$. Hence $f(x), f(y) \in f(C)$ and $f(x) \neq f(y)$, while $f(z) \in C_{\infty}$. Now the capacity of $F$ is preserved under a similarity transformation $T$. With $\tilde{f}=T \circ f$ we assume that $\tilde{f}(x)=0$ and $\tilde{f}(y)=(-1,0, \ldots, 0)=-e_{1}$. Next let $\tilde{f}(C)^{*}$ be the spherical symmetrization of $\tilde{f}(C)$ about the negative $x_{1}$-axis and $\tilde{f}(B)^{*}=R^{n} \backslash D$ where $D$ is the spherical symmetrization of $R^{n} \backslash \tilde{f}(B)$ in the positive $x_{1}$-axis. By Theorem 5.2

$$
\operatorname{cap}\left(\tilde{f}(B)^{*}, \tilde{f}(C)^{*}\right) \leq \operatorname{cap}(\tilde{f}(B), \tilde{f}(C)) .
$$

We denote by $R_{T}(t)$ the Teichmüller condenser $\left(R^{n} \backslash L_{2}, L_{1}\right)$ where $L_{1}$ is the line segment from $-e_{1}$ to 0 and $L_{2}$ is the ray on the $x_{1}$-axis from $t e_{1}$ to $\infty$. If $t=|\tilde{f}(z)|$, then $L_{1} \subset \tilde{f}(C)^{*}$ and $\tilde{f}(B)^{*} \subset R^{n} \backslash L_{2}$. Hence by (5.1)

$$
\operatorname{cap} R_{T}(|\tilde{f}(z)|) \leq \operatorname{cap}\left(\tilde{f}(B)^{*}, \tilde{f}(C)^{*}\right) \text {. }
$$

We also have the following lower bound for the capacity of the Teichmüller condenser (see [6, p. 518, Lemma 8 and 20, p. 89, Lemma 7.22]).

$$
\operatorname{cap} R_{T}(t) \geq \omega_{n-1}[\log \lambda(t+1)]^{1-n} \text {. }
$$

Here $\lambda$ is a constant which depends only on $n$. Moreover since $f$ is $K$ quasiregular in $\Omega$

$$
\operatorname{cap} F \leq K \operatorname{cap} E
$$

[12, p. 29, Theorem 7.1]. Notice that $|\tilde{f}(z)|=|f(z)-f(x)| /|f(y)-f(x)|$. Combining (5.6), (5.5), (5.4), (5.7), and (5.3) we obtain

$$
\begin{gathered}
\omega_{n-1}\left[\log \lambda\left(1+\frac{|f(z)-f(x)|}{|f(y)-f(x)|}\right)\right]^{1-n} \leq \operatorname{cap}(\tilde{f}(B), \tilde{f}(C)) \\
=\operatorname{cap}(f(B), f(C)) \leq K \omega_{n-1}\left[\log \frac{d(x, \partial \Omega)}{2|x-y|}\right]^{1-n} .
\end{gathered}
$$

Next choose $x_{0} \in \partial \Omega$ such that $\left|x-x_{0}\right|=d(x, \partial \Omega)$. We have the estimate

$$
\begin{aligned}
\mid f(y) & -f(x)|+| f(z)-f(x) \mid \\
& \leq C_{1}\left(2\left|x_{0}-x\right|^{\alpha}+\left|z-x_{0}\right|^{\alpha}+\left|y-x_{0}\right|^{\alpha}\right) \\
& \leq 6 C_{1} d(x, \partial \Omega)^{\alpha} .
\end{aligned}
$$

Using (5.9) we can rewrite (5.8),

$$
|f(x)-f(y)| \leq 6 \lambda C_{1} 2^{\alpha^{\prime}} d(x, \partial \Omega)^{\alpha-\alpha^{\prime}}|x-y|^{\alpha^{\prime}}
$$

where $\alpha^{\prime}=K^{1 /(1-n)}$. If $\alpha \leq \alpha^{\prime}$, then since $|x-y|<d(x, \partial \Omega) / 2$ we have $f \in$ $\operatorname{Lip}_{\alpha}(\bar{\Omega})$. Otherwise, if $\Omega$ is bounded, then $f \in \operatorname{Lip}_{\alpha^{\prime}}(\Omega)$ since $d(x, \partial \Omega) \leq$ Euclidean diameter of $\Omega$.

Theorem 5.3 also gives the following. 
5.4. Corollary. If $f$ is $K$-quasiregular in a bounded domain $\Omega \subset R^{n}$ and if (1.5) is satisfied for all $x \in \Omega$ with $0<\alpha \leq 1$, then $f \in \operatorname{Lip}_{\beta}(\bar{\Omega})$ where $\beta=\min \left(\alpha, K^{1 /(1-n)}\right)$.

5.5. Remark. Because the components of a quasiregular mapping satisfy $\left|\nabla f_{i}\right|$ $\leq K\left|\nabla f_{j}\right|$ a.e., Theorem 1.3 implies a special case of the main result in [14, $\mathrm{p}$. 705, Theorem 3.8]. Namely the components belong to the same Lipschitz class.

6.1. Bounded mean oscillation. For $f: \Omega \rightarrow R^{m}$ we denote the BMO-norm by

$$
\|f\|_{*}=\sup _{B \subset \Omega} \frac{1}{|B|} \int_{B}\left|f-f_{B}\right| \text {. }
$$

Here the supremum is taken over all balls $B \subset \Omega$ and $f_{B}$ is the average value of $f$ over $B,(1 /|B|) \int_{B} f$. In the case that $u$ is harmonic in $\Omega,\|u\|_{*}<\infty$ if and only if $|\nabla u(x)| \leq C d(x, \partial \Omega)^{-1}$ for all $x \in \Omega$ and consequently, if and only if $D_{u}(x) \leq C d(x, \partial \Omega)^{-1}$ for all $x \in \Omega$. Theorem 1.4 is a generalization of this result. Theorem 6.2 is a special case of the main result in ([18], see inequality (1.4)). In the case that $\Omega$ is a half space, it appears in [16, p. 4, Hilfssatz 2].

6.2. Theorem. If $f: \Omega \rightarrow R^{m}$ satisfies

$$
\sup _{2 B \subset \Omega} \frac{1}{|B|} \int_{B}\left|f-f_{B}\right|<\infty
$$

where the supremum is taken over all balls $B$ with $2 B \subset \Omega$, then $\|f\|_{*}<\infty$.

The next result follows from the John-Nirenberg Theorem [5, p. 233, Corollary 2.3 and 16, p. 32].

6.3. Theorem. If $f: \Omega \rightarrow R^{m}$, and if $1 \leq p<\infty$, then there exists a constant $C$, depending only on $n$ and $p$, such that

$$
\left(\frac{1}{|B|} \int_{B}\left|f-f_{B}\right|^{p}\right)^{1 / p} \leq C\|f\|_{*}
$$

for all balls $B \subset \Omega$.

6.4. Proof of Theorem 1.4. Assume that (1.5) holds for all $x \in \Omega$. By Theorem 6.2 it is sufficient to show that $f$ satisfies (6.1). Let $x_{0} \in \Omega$ and $B=B\left(x_{0}, \frac{1}{2} d\left(x_{0}, \partial \Omega\right)\right)$. Using Hölder's inequality and (2.2) we obtain

$$
\frac{1}{|B|} \int_{B}\left|f_{j}-\left(f_{j}\right)_{B}\right| \leq\left(\frac{1}{|B|} \int_{B}\left|f_{j}-\left(f_{j}\right)_{B}\right|^{n}\right)^{1 / n} \leq 2 \operatorname{diam} B D_{f_{j}}\left(x_{0}\right) \text {. }
$$

Since $\operatorname{diam} B=d\left(x_{0}, \partial \Omega\right),(1.7)$ gives

$$
\frac{1}{|B|} \int_{B}\left|f_{j}-\left(f_{j}\right)_{B}\right| \leq 2 C_{1}
$$

and (6.1) follows.

Next assume that (1.8) holds. Applying (2.4) with $\sigma=2$,

$$
\left(\frac{1}{|B|} \int_{B}\left|\nabla f_{j}\right|^{n}\right)^{1 / n} \leq \frac{C(n, K)}{\operatorname{diam} B}\left(\frac{1}{|2 B|} \int_{2 B}\left|f_{j}-a\right|^{n}\right)^{1 / n}
$$


for all $a \in R$. Choosing $a=\left(f_{j}\right)_{2 B}$ in (6.3) and $p=n$ in (6.2) we obtain

$$
D_{f_{j}}\left(x_{0}\right) \leq \frac{C(n, K)\|f\|_{*}}{d\left(x_{0}, \partial \Omega\right)} \leq C_{2} C(n, K) d\left(x_{0}, \partial \Omega\right)^{-1}
$$

Theorem 1.4 provides another proof of the following result which appears in [10, p. 280, Corollary 3].

6.5. Corollary. If $f=\left(f_{1}, f_{2}, \ldots, f_{n}\right)$ is $K$-quasiregular in $\Omega$, then the BMOnorms $\left\|f_{j}\right\|_{*}, j=1,2, \ldots, n$, are equivalent.

Proof. The operators $D_{f_{j}}(x)$ are equivalent since $\left|\nabla f_{j}(x)\right| \leq K\left|\nabla f_{i}(x)\right|$ a.e. for all $i, j=1,2, \ldots, n$.

\section{REFERENCES}

1. K. Astala and F. W. Gehring, Quasiconformal analogues of theorems of Koebe and Hardy and Littlewood, Michigan Math. J. 32 (1985), 99-108.

2. _ Injectivity, the BMO norm and the universal Teichmüller space, J. Analyse Math. 46 (1986), 16-57.

3. B. Bojarski and T. Iwaniec, Analytical foundations of the theory of quasiconformal mappings in $R^{n}$, Ann. Acad. Sci. Fenn. Ser. A I Math. 8 (1983), 257-324.

4. P. L. Duren, Theory of $H^{p}$-spaces, Academic Press, 1970.

5. J. B. Garnett, Bounded analytic functions, Academic Press, 1981.

6. F. W. Gehring, Symmetrization of rings in space, Trans. Amer. Math. Soc. 101 (1961), 499-519.

7. $\ldots$, The $L^{p}$-integrability of the partial derivatives of a quasiconformal mapping, Acta Math. 130 (1973), 265-277.

8. F. W. Gehring and O. Martio, Lipschitz classes and quasiconformal mappings, Ann. Acad. Sci. Fenn. Ser. A I Math. 10 (1985), 203-219.

9. G. H. Hardy and J. E. Littlewood, Some properties of fractional integrals. II, Math. Z. 34 (1932), 403-439.

10. T. Iwaniec and C. A. Nolder, Hardy-Littlewood inequality for quasiregular mappings in certain domains in $R^{n}$, Ann. Acad. Sci. Fenn. Ser. A I Math. 10 (1985), 267-282.

11. V. Lappalainen, Lip ${ }_{h}$-extension domains, Ann. Acad. Sci. Fenn. Ser. A I Math. Dissertationes 56 (1985).

12. O. Martio, S. Rickman and J. Väisälä, Definitions for quasiregular mappings, Ann. Acad. Sci. Fenn. Ser. A I Math. 448 (1969), 1-40.

13. R. Näkki and B. Palka, Lipschitz conditions and quasiconformal mappings, Indiana Univ. Math. J. 31 (1982), 377-401.

14. C. A. Nolder, Conjugate functions and moduli of continuity, Illinois J. Math. 31 (1987), 699-709.

15. H. M. Reimann, Functions of bounded mean oscillation and quasiconformal mappings, Comment. Math. Helv. 49 (1974), 260-276.

16. H. M. Reimann and T. Rychener, Funktionen beschränkter mittlerer Oszillation, Lecture Notes in Math., vol. 487, Springer-Verlag, 1975.

17. J. Sarvas, Symmetrization of condensers in $n$-space, Ann. Acad. Sci. Fenn. Ser. A I Math. 522 (1972), 1-44.

18. S. Staples, $L^{p}$-averaging domains and the Poincaré inequality, Ann. Acad. Sci. Fenn. Ser. A I Math. 14 (1989), 103-127.

19. M. Vuorinen, Conformal invariants and quasiregular mappings, J. Analyse Math. 45 (1985), 69-115.

20. __ Conformal geometry and quasiregular mappings, Springer-Verlag, 1988.

Department of Mathematics, Florida State University, Tallahassee, Florida 32306 - 